\title{
BMJ
}

\section{Cost effectiveness of management strategies for urinary tract infections: results from randomised controlled trial}

\author{
David Turner, principal research fellow in health economics, ${ }^{1}$ Paul Little, professor of primary care research, ${ }^{2}$ \\ James Raftery, professor, director of NETSCC and Wessex Institute, ${ }^{1}$ Sheila Turner, senior research fellow, ${ }^{1}$ \\ Helen Smith, chair of primary care, ${ }^{3}$ Kate Rumsby, research programme manager, Wessex Research \\ Practices Collaboration, ${ }^{2}$ Mark Mullee, director of the Research Design Service South Central and senior \\ lecturer in medical statistics, ${ }^{2}$ on behalf of the UTIS team
}

\section{${ }^{1}$ Wessex Institute, University of Southampton, Alpha House, Southampton Science Park, Chilworth, Southampton S016 7NS \\ ${ }^{2}$ Community Clinical Sciences Division, Southampton University, Southampton S016 6ST \\ ${ }^{3}$ Division of Public Health and Primary Care, Brighton and Sussex Medical School, Brighton Correspondence to: $\mathrm{D}$ Turner dturner@soton.ac.uk}

Cite this as: BMJ 2010;340:c346 doi:10.1136/bmj.c346

\section{ABSTRACT}

Objective To assess the cost effectiveness of different management strategies for urinary tract infections. Design Cost effectiveness analysis alongside a randomised controlled trial with a one month follow-up. Setting Primary care.

Participants 309 non-pregnant adult women aged 18-70 presenting with suspected urinary tract infection. Interventions Patients were randomised to five basic management approaches: empirical antibiotics, empirical delayed (by 48 hours) antibiotics, or targeted antibiotics based on either a high symptom score (two or more of urine cloudiness, smell, nocturia, dysuria), dipstick results (nitrite or leucocytes and blood), or receipt of a positive result on midstream urine analysis. Main outcome measure Duration of symptoms and cost of care.

Results Management with targeted antibiotics with midstream urine analysis was more costly over the period of one month. Costs for the midstream urine analysis and dipstick management groups were $£ 37$ and $£ 35$, respectively; these compared with $£ 31$ for immediate antibiotics. Cost effectiveness acceptability curves suggested that if avoiding a day of moderately bad symptoms was valued at less than $£ 10$, then immediate antibiotics is likely to be the most cost effective strategy For values over $£ 10$, targeted antibiotics with dipstick testing becomes the most cost effective strategy, though because of the uncertainty we can never be more than $70 \%$ certain that this strategy truly is the most cost effective.

Conclusion Dipstick testing with targeted antibiotics is likely to be cost effective if the value of saving a day of moderately bad symptoms is $£ 10$ or more, but caution is required given the considerable uncertainty surrounding the estimates.

\section{INTRODUCTION}

Urinary tract infections are common in female patients in general practice, ${ }^{1}$ and urine is the most frequently received specimen in microbiology laboratories. These common bacterial infections affect half of all women at least once in their lives. ${ }^{2}$ Clinical management strategies vary, with empirical antibiotics commonly used. Many practitioners, however, also use urinary dipstick results and request analysis of midstream urine samples. In over $62 \%$ of women presenting with suspected urinary tract infection, the diagnosis is laboratory confirmed. ${ }^{3}$ The use of dipsticks and clinical scoring algorithms can potentially help to improve the precision of diagnosis by improving the positive predictive values. ${ }^{3}$ It is unclear what effects different management strategies will have on the costs and cost effectiveness. We carried out an economic evaluation alongside a randomised controlled trial to investigate the costs and cost effectiveness of the different management strategies for urinary tract infection.

\section{METHODS}

Randomised controlled trial

General practitioners or practice nurses recruited patients with suspected urinary tract infection, either first time or recurrent, on presentation. The study included 309 non-pregnant adult women aged 18-70. The practitioner collected baseline symptoms, clinical information, and demographic details. Patients completed a symptom diary for 14 days. Patients were randomised to one of five management groups: empirical antibiotic treatment; empirical delayed antibiotics; antibiotic targeted by symptom score; antibiotic according to dipstick algorithm (nitrites or leucocytes and a trace of blood); or midstream urine analysis with symptomatic treatment until culture and sensitivity results were available and then antibiotics targeted accordingly. Full details of this trial are given elsewhere. ${ }^{4}$

\section{Costs}

Costs were estimated from an NHS perspective and included the recruitment consultation with the general practitioner and all NHS contacts related to urinary tract infection in a one month follow-up period. Costs for the recruitment visit included the consumable costs for midstream urine analysis and dipstick tests plus 
Table 1 |Resource use by patients* with urinary tract infection according to allocation to management strategy group

\begin{tabular}{|c|c|c|c|c|c|}
\hline & $\begin{array}{l}\text { Immediate antibiotics } \\
\qquad(n=56)\end{array}$ & $\begin{array}{l}\text { Midstream urine } \\
\qquad(\mathrm{n}=46)\end{array}$ & $\begin{array}{l}\text { Dipstick } \\
(n=42)\end{array}$ & $\begin{array}{l}\text { Symptom scores } \\
\qquad(n=60)\end{array}$ & $\begin{array}{l}\text { Delayed antibiotics } \\
\qquad(\mathrm{n}=53)\end{array}$ \\
\hline \multicolumn{6}{|c|}{ Recruitment visit to general practice } \\
\hline Mean length (mins) & 11.6 & 12.4 & 13.0 & 11.9 & 12.5 \\
\hline Midstream urine samples taken & 15 & 40 & 16 & 20 & 8 \\
\hline Dipstick tests carried out & 31 & 22 & 40 & 35 & 17 \\
\hline No (\%) prescribed antibiotics & $54(96)$ & $38(83)$ & $32(76)$ & $52(87)$ & $40(75)$ \\
\hline \multicolumn{6}{|c|}{ Reconsultations with general practitioner within 1 month } \\
\hline No of visits & 6 & 9 & 6 & 8 & 6 \\
\hline Midstream urine sample taken & 3 & 3 & 4 & 5 & 3 \\
\hline Prescribed antibiotics & 4 & 7 & 5 & 7 & 5 \\
\hline Referred to secondary care & 0 & 0 & 0 & 0 & 0 \\
\hline
\end{tabular}

laboratory costs for the urine analysis. Consumable and laboratory costs were those of the local NHS trust. The recruitment consultation was costed with national unit costs per minute based on the duration of consultation. ${ }^{5}$ The time taken to explain and perform the test was included within this consultation.

As we had no data on length of consultation for $39 / 309$ patients $(13 \%)$ and antibiotic use for $41 / 309$ (13\%), we imputed missing values using maximum likelihood estimation for consultation time and a regression method (with results constrained to be yes or no) for antibiotic use (SPSS version 14). Original data were checked to ensure that the means and standard errors of the estimates for the imputed variables were equivalent (mean values within 1\%) to those of the originals. These two items were imputed as the data relied on completion by the practitioner and we had no a priori reason that it would be correlated with patients' characteristics and so would be unlikely to bias results. Not imputing would have lost an additional 49 cases for the final analysis. For costing purposes we assumed that antibiotics were prescribed according to the study protocol - that is, trimethoprim, costed as in the British National Formulary. ${ }^{6} \mathrm{We}$ obtained details of use of resources during follow-up directly from the patient's general practice notes. Contacts with general practitioners during follow-up were costed as a standard 10 minute consultation. ${ }^{5}$ Midstream urine analysis and antibiotic use were recorded and costed with methods described earlier. Referrals to secondary care during follow-up were included if there was a reasonable probability of the referral being attributable to urinary tract infections; this was done in consultation with a clinical expert blind to management strategy (PL). We used NHS reference costs. ${ }^{7}$ All costs were estimated for the year 2005-6 in pounds (£). No time discounting was required.

\section{Analysis}

Using Microsoft Excel and SPSS version 14, we analysed the $83 \%(257 / 309)$ of participants for whom we had estimates of both effectiveness and follow-up costs. The economic evaluation was a cost effectiveness study in terms of cost per day of moderate or severe symptoms avoided. Symptom days were obtained from the patient's diary completed for the 14 days after recruitment. To test the robustness of estimates obtained we also estimated means using 1000 bootstrap samples. ${ }^{89}$ Bootstrapping was also used to estimate cost effectiveness acceptability curves (CEAC) for the cost per day of moderate or severe symptoms avoided. ${ }^{10}$

\section{Scenario analysis}

To investigate the potential cost per QALY of different management strategies a scenario analysis used a utility loss of 0.2894 for a day with urinary tract infection symptoms. ${ }^{11} \mathrm{We}$ also carried out a threshold analysis to show the utility loss per day of symptoms required to produce a cost per QALY ratio of between $£ 20000$ and $£ 30000$, the values used in NICE (National Institute for Health and Clinical Excellence) decision making. ${ }^{12}$

\section{RESULTS}

The resources used by patients in the five management groups varied as expected (table 1), with those in the dipstick group requiring the longest consultation and those in the empirical antibiotics group the shortest. The frequency of antibiotic use and investigations also varied as expected between management groups, as did the costs at one month (range £30.70-£37.10) (table 2). These costs were attributable mainly to the initial consultation.

Management strategies were ranked by mean effectiveness (table 3); differences between groups were small. Three strategies were dominated (delayed antibiotics, symptom score, and midstream urine), meaning some other strategy was both less costly and more effective. The immediate antibiotics and dipstick groups were not dominated and incremental results are given for the dipstick group compared with the immediate antibiotic group. Although no effect differences were significant in conventional (frequentist) statistics, current health economic practices combine such data in cost effectiveness acceptability curves.

Cost effectiveness acceptability curves by strategy (figure) indicate that if avoiding a day of symptoms is valued at less than $£ 10$, the most cost effective strategy 
Table $2 \mid$ Mean (SD) costs ( $£$ ) and effectiveness of management strategies for urinary tract infection

\begin{tabular}{|c|c|c|c|c|c|}
\hline & Immediate antibiotics & Midstream urine & Dipstick & Symptom scores & Delayed antibiotics \\
\hline \multicolumn{6}{|l|}{ Recruitment visit: } \\
\hline General practice visit & $25.5(8.7)$ & $27.3(11)$ & $28.6(9.5)$ & $26.2(10.3)$ & $27.4(10.8)$ \\
\hline Midstream urine & $1.2(1.9)$ & $3.8(1.5)$ & $1.7(2.1)$ & $1.4(2.1)$ & $0.7(1.6)$ \\
\hline Dipstick & $0.2(0.2)$ & $0.2(0.2)$ & $0.4(0.1)$ & $0.3(0.2)$ & $0.1(0.2)$ \\
\hline Antibiotics & $1.0(0.2)$ & $0.8(0.4)$ & $0.8(0.4)$ & $0.9(0.3)$ & $0.7(0.4)$ \\
\hline Total & $27.8(9.1)$ & $32.1(11.1)$ & $31.4(9.8)$ & $28.7(10.2)$ & $29.0(11.2)$ \\
\hline \multicolumn{6}{|l|}{ Follow-up at 1 month: } \\
\hline General practice visit & $2.4(6.9)$ & $4.3(8.8)$ & $3.1(9.2)$ & $2.9(8.6)$ & $2.5(8.3)$ \\
\hline Midstream urine & $0.2(1)$ & $0.3(1.1)$ & $0.4(1.3)$ & $0.4(1.2)$ & $0.2(1)$ \\
\hline Referred to secondary care & $0(0)$ & $0(0)$ & $0(0)$ & $0(0)$ & $0(0)$ \\
\hline Antibiotics & $0.2(0.9)$ & $0.4(1.4)$ & $0.3(1)$ & $0.2(0.8)$ & $0.1(0.4)$ \\
\hline Total & $2.8(8.2)$ & $5.0(10.4)$ & $3.9(11)$ & $3.5(10.2)$ & $2.9(9.5)$ \\
\hline Total cost in 1st month & $30.6(13.9)$ & 37.1 (15) & 35.3 (13.3) & 32.3 (13.9) & 31.9 (15.8) \\
\hline $\begin{array}{l}\text { Effectiveness (days of } \\
\text { moderate/severe symptoms) }\end{array}$ & $3.63(2.7)$ & 4.17 (3.1) & $3.14(2.1)$ & $3.92(3.6)$ & $3.92(3.8)$ \\
\hline
\end{tabular}

is likely to be immediate antibiotics. For values over $£ 10$, the dipstick strategy becomes the most cost effective, although given the uncertainty present we can only ever be $70 \%$ certain that this strategy really is the most cost effective. The scenario analysis produced an incremental cost per QALY ratio of $£ 12100$ for the dipstick test compared with immediate antibiotics. Threshold analysis indicated that a utility loss per day of symptoms of between 0.117 and 0.175 produced a cost per QALY of between $£ 20000$ and $£ 30000$.

\section{DISCUSSION}

All management strategies for urinary tract infection have similar resource implications. The similarities in cost are not surprising as the tests used in this study have low costs and would entail only small changes to the time practitioners would need to provide care for a patient with a urinary tract infection. Therefore there is no strong reason to prefer any particular management strategy on the basis of costs. The duration of symptoms was similar between groups, with the dipstick strategy being associated with the shortest duration of symptoms. Compared with the group given immediate antibiotics, the dipstick strategy cost $£ 10$ per additional day of symptoms avoided.

\section{Strengths and limitations}

The current study is based on a randomised controlled trial with a rigorously developed dipstick algorithm and clinical score. The studied group comprised women in whom urinary tract infection was the suspected diagnosis. ${ }^{13}$ They had similar characteristics to national attending samples in the United Kingdom ${ }^{1}$ so study results should be representative of routine clinical practice. Data on resource was obtained from study records or from general practitioners' notes.

The cost effectiveness analysis shown here presents estimates of the cost per day of symptoms avoided. These results should be interpreted with caution because of the lack of significance in the effectiveness data. ${ }^{4}$ It is unclear why there were better outcomes in the dipstick group than in the immediate group as use of antibiotics was higher in the immediate group $(96 \% v$ $76 \%$ ). Those in the dipstick group, however, had less severe symptoms. ${ }^{4}$ The improvement in the dipstick group could be related to the security of feeling that they know from dipsticks that they do or do not have an infection.

We considered only costs related to the NHS; this might miss important costs such as travel and time off work, which might be relevant as $83 \%$ of the participants were aged under 60 . We had details on time off work or study for 146/257 women and of these 48/146 (33\%) needed time off because of their infection. There were no significant differences, however, between any of the groups so it is unlikely that considering these costs would have altered the results. Some additional travel costs would have been associated with reconsultations but these were low, with an average of 0.13 reconsultations during follow-up.

Our analysis excluded any benefits associated with reducing the use of antibiotics, such as less antibiotic resistance. Strategies that reduce use of antibiotics are thus undervalued. ${ }^{14}$ The highest numbers prescribed antibiotics were in the group prescribed immediate antibiotics (table 1).

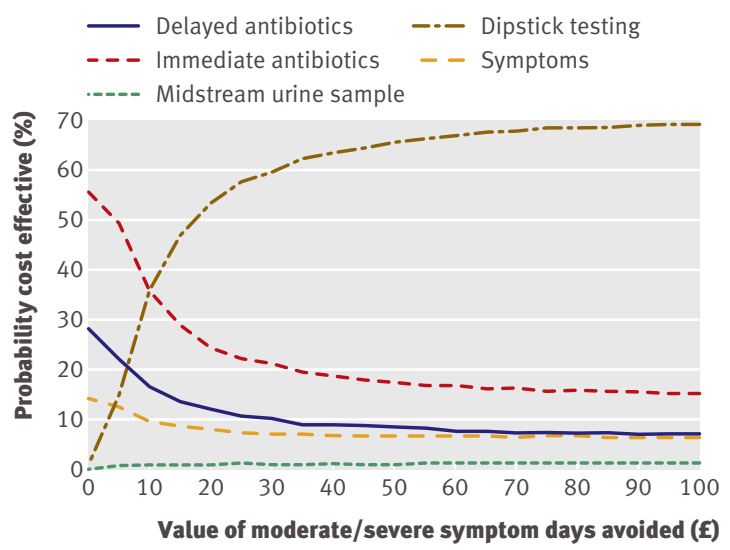

$\overline{\text { Cost effectiveness acceptability curve for one month follow-up }}$ 
Table $3 \mid$ Cost effectiveness of strategies at one month follow-up

\begin{tabular}{|c|c|c|c|c|c|}
\hline Study group & Total cost & $\begin{array}{l}\text { Days of moderate/ } \\
\text { severe symptoms }\end{array}$ & Incremental costs & $\begin{array}{l}\text { Incremental days of } \\
\text { symptoms avoided* }\end{array}$ & $\begin{array}{l}\text { Incremental cost } \\
\text { effectiveness/day of } \\
\text { symptom avoided }\end{array}$ \\
\hline Midstream urine & $£ 37$ & 4.17 & Dominated & Dominated & Dominated \\
\hline Delayed & $£ 32$ & 3.92 & Dominated & Dominated & Dominated \\
\hline Symptom score & $£ 32$ & 3.92 & Dominated & Dominated & Dominated \\
\hline Immediate & $£ 31$ & 3.63 & - & - & - \\
\hline Dipstick† & $£ 35$ & 3.14 & $£ 5$ & 0.48 & $£ 9.70$ \\
\hline
\end{tabular}

*Incremental symptom days avoided calculated as difference in numbers of symptom days compared with next best alternative.

†Dipstick group compared with immediate group.

\section{Comparison with other studies}

There is limited evidence on the cost effectiveness of different management strategies for urinary tract infection in primary care. Three studies used modelling to examine similar questions. The literature identified is either from the United States or the UK and hence the values presented here use different currency unit and cost years. To aid comparison we also present values in 2005- $6 £$ using $\mathrm{UK}^{15}$ or US ${ }^{16}$ inflation indicators and an exchange rate of $\$ 1.78$ to the $£^{17}$; adjusted values are given in brackets. All the studies found that immediate (empirical) antibiotics were the least costly. A UK based study of management of urinary tract infection in general practice found that laboratory testing with empirical antibiotics resulted in earlier resolution of symptoms at a cost of $£ 215$ ( $£ 246$ ) per day of symptoms avoided compared with empirical antibiotics. ${ }^{18}$ A US cost utility study found that compared with empirical treatment a complete urinalysis cost $\$ 2964$ per additional QALM (quality adjusted life month) and dipsticks cost \$48 460 per QALM (equivalent to $£ 25682$ and $£ 419894$ per QALY, respectively). ${ }^{11} \mathrm{~A}$ further study found that treatment for urinary tract infection with accompanying over-the-counter dipstick test cost between $\$ 5.50$ and $\$ 1.97$ ( $£ 4.14$ and $£ 1.48$ ) per day of symptoms avoided, depending on how effective the dipstick was in directing treatment to those who actually had a urinary tract infection. ${ }^{14}$ These results are consistent with those presented here as they show that tests can be associated with additional costs and additional days of symptoms avoided.

\section{Interpretation of results}

Our analysis suggests that each additional day of symptoms can be avoided at a cost of about $£ 10$. A judgment

\section{WHAT IS ALREADY KNOWN ON THIS TOPIC}

Urinary tract infections are a common reason for consultation in primary care

There is limited information on the economic implications of common management strategies for these infections in primary care

\section{WHAT THIS STUDY ADDS}

Dipstick testing with targeted antibiotics is likely to be cost effective if the value of saving a day of moderately bad symptoms is valued at $£ 10$ or more

The cost effectiveness of strategies depends on the value placed on days of moderate or severe symptoms avoided would therefore need to be made of whether the value of avoiding days of moderate or severe symptoms would exceed this. The scenario analysis we constructed suggested that if a day of symptoms was associated with a utility loss of 0.2984 then the dipstick strategy could generate extra QALYs at a cost per QALY of around $£ 12000$. This analysis should be treated with caution, however, as the estimate of 0.2984 was not derived directly from patients. More information on utility losses associated with urinary tract infection symptoms would be important in determining the value of days of symptoms avoided and hence in informing the care of affected patients.

We thank the practitioners for their time and Bayer for provision of the 8SG strips.

Contributors: PL had the original ideas for the study. ST ran the clinical trial on a day to day basis. KR managed the database. DT carried out the economic analysis in consultation with JR, PL, and MM. PL and HS contributed clinical knowledge. MM advised on statistical matters. All authors contributed to writing the paper. DT is guarantor.

Funding: This study was funded by the Health Technology Programme of UK NHS Research and Development.

Competing interests: All authors have completed the Unified Competing Interest form at www.icmje.org/coi_disclosure.pdf (available on reques from the corresponding author) and declare (1) no support from any company for the submitted work; (2) no relationships with any companies that might have an interest in the submitted work in the previous 3 years; (3) their spouses, partners, or children have no financial relationships that may be relevant to the submitted work; and (4) no nonfinancial interests that may be relevant to the submitted work.

Ethical approval: This study was approved by the South West MReC ethical committee and informed consent was given by all patients. Data sharing: No additional data available.

1 HMSO, OPCS. Morbidity statistics from general practice: fourth national study 1991. HMSO, 1991.

2 Foxman B. Epidemiology of urinary tract infections: incidence, morbidity, and economic costs. Dis Mon 2003;49:53-70.

3 Little P, Turner S, Rumsby K, Warner G, Moore M, Lowes JA, et al. Developing clinical rules to predict urinary tract infection in primary care settings: sensitivity and specificity of near patient tests (dipsticks) and clinical scores. Br J Gen Pract 2006;56:606-12.

4 Little P, Moore M, Turner S, Rumsby K, Warner G, Lowes A, et al. Effectiveness of five different approaches in the management of urinary tract infection: a randomised controlled trial. $B M$ J 2010 doi:10.1136/bmj.c199.

5 Curtis L, Netten A. Unit costs of health and social care. University of Kent, 2006. http://www.pssru.ac.uk/uc/uc2006contents.htm.

6 British Medical Association. British National Formulary (BNF). Number 51. BMJ Group and RPS Publishing, 2006.

7 Department of Health. NHS reference costs 2005-06. 2010. www.dh. gov.uk/en/Publicationsandstatistics/Publications/ PublicationsPolicyAndGuidance/DH 062884.

8 Barber JA, Thompson SG. Analysis of cost data in randomized trials: an application of the non-parametric bootstrap. Stat Med 2000;19:3219-36.

9 Thompson SG, Barber JA. How should cost data in pragmatic randomised trials be analysed? BMJ 2000;320:1197-200. 
10 Löthgren M, Zethraeus N. Definition, interpretation and calculation of cost-effectiveness acceptability curves. Health Econ 2000;9:623-30.

11 Barry HC, Ebell MH, Hickner J. Evaluation of suspected urinary tract infection in ambulatory women: a cost-utility analysis of office-based strategies. J Fam Pract 1997;44:49-60.

12 National Institute for Health and Clinical Excellence (NICE). Guide to the methods of technology appraisal. 2009. www.nice.org.uk/ aboutnice/howwework/devnicetech/

technologyappraisalprocessguides/

guidetothemethodsoftechnologyappraisal.jsp?

domedia=1\&mid=B52851A3-19B9-E0B5-D48284D172BD8459.

13 Bent S, Nallamothu B, Simel D, Finn S, Saint S. Does this woman have an acute uncomplicated urinary tract infection? JAMA 2002;287:2701-10.
14 Rubin N, Foxman B. The cost-effectiveness of placing urinary tract infection treatment over the counter. J Clinl Epidemiol 1996;49:1315-21.

15 Office for National Statistics. Retail prices index: monthly index numbers of retail prices 1948-2008 (RPI) (RPIX). 2010. www. statistics.gov.uk/STATBASE/tsdataset.asp?vlnk=229.

16 Bureau of Labor Statistics. CPI detailed report: data for April 2008. 2008. www.bls.gov/cpi/cpid0804.pdf.

17 BBC. Market data. 2010. http://newsvote.bbc.co.uk/1/shared/fds/ hi/business/market_data/currency/default.stm.

18 Fenwick EA, Briggs AH, Hawke $\mathrm{Cl}$. Management of urinary tract infection in general practice: a cost-effectiveness analysis. Br J Gen Pract 2000;50:635-9.

Accepted: 18 December 2009 